\title{
THE PROBLEM OF PLATEAU $\dagger$
}

\author{
BY JESSE DOUGLAS
}

1. Formulation. The problem of Plateau is to prove the existence of a minimal surface bounded by a given contour. The first and only complete solution of this problem was found by the present author $[1-11] \ddagger$; in this solution the contour is an arbitrary Jordan curve in $n$-dimensional euclidean space. Besides this generality of result, the chief contribution of the work seems to lie in the introduction of a certain new functional $A(g)$, which furnishes the key to the Plateau problem and makes clear its relationship to other fundamental problems of analysis.

For definition of minimal surface, we take the formulas of Weierstrass,

$$
x_{i}=\Re F_{i}(w), \quad \sum_{i=1}^{n} F_{i}^{\prime 2}(w)=0
$$

though Weierstrass himself considered only the case $n=3$. Here $w$ is a complex variable, $w=u+i v$, of which the $F_{i}$ are monogenic functions. When $n=2$, these formulas become

$$
x_{1}=\Re F_{1}(w), x_{2}=\Re F_{2}(w), F_{1}^{\prime 2}(w)+F_{2}^{\prime 2}(w)=0,
$$

or

$$
F_{2}^{\prime}(w) \pm i F_{1}^{\prime}(w)=0
$$

If

$$
F_{1}(w)=P_{1}(u, v)+i Q_{1}(u, v), \quad F_{2}(w)=P_{2}(u, v)+i Q_{2}(u, v),
$$

then

$$
\begin{aligned}
& F_{1}^{\prime}(w)=\frac{\partial P_{1}}{\partial u}+i \frac{\partial Q_{1}}{\partial u}=\frac{\partial P_{1}}{\partial u}-i \frac{\partial P_{1}}{\partial v} \\
& F_{2}^{\prime}(w)=\frac{\partial P_{2}}{\partial u}+i \frac{\partial Q_{2}}{\partial u}=\frac{\partial P_{2}}{\partial u}-i \frac{\partial P_{2}}{\partial v}
\end{aligned}
$$

$\dagger$ Address delivered by invitation of the program committee at the New York meeting, October 29, 1932.

$\ddagger$ Numerical references are to the bibliography at the end. 
where we have used the Cauchy-Riemann equations for $P_{1}, Q_{1}$, and $P_{2}, Q_{2}$. If we use the plus sign, $\dagger(3)$ implies that

$$
\frac{\partial P_{1}}{\partial u}=\frac{\partial P_{2}}{\partial v}, \quad \frac{\partial P_{1}}{\partial v}=-\frac{\partial P_{2}}{\partial u},
$$

which are the Cauchy-Riemann equations for the functions (2), that is, these functions are conjugate harmonic. The equations (2) therefore represent a conformal map of the plane $x_{1}+i x_{2}$ on the $w$ plane.

Consequently the problem of Plateau, as I have formulated and solved it, contains the conformal mapping problem of Riemann: to map conformally a given plane region on the interior of a circle (in the very general case of any region bounded by a Jordan curve). It will include also the theorem of Osgood [31] and Carathéodory [32] to the effect that the map remains one-to-one and continuous as between the circumference of the unit circle and the Jordan curve bounding the given region.

2. Historical. The Plateau problem is named for the Belgian natural scientist J. Plateau [27], who made numerous experiments with soap films, realizing a large variety of minimal surfaces. A soap film, under its surface tension, takes the form of least area consistent with its constraints, that is, with the condition of being bounded by a given contour; and this least area property is a characteristic of minimal surfaces.

It was in this way, indeed, that minimal surfaces first appeared in mathematics, in a pioneer memoir of Lagrange (1760) [28] on the calculus of variations for double integrals. The analytic formulation of the problem of least area is

$$
\iint\left(1+p^{2}+q^{2}\right)^{1 / 2} d x d y=\text { minimum, }
$$

among all surfaces $z=f(x, y)$ with a given boundary $\Gamma$, and the condition found by Lagrange for the minimizing surface is

$$
\left(1+q^{2}\right) r-2 p q s+\left(1+p^{2}\right) t=0,
$$

where $p, q$, and $r, s, t$ denote, as usual, the first and second partial derivatives of $z$. Some years later (1776), Meusnier in-

$\dagger$ The minus sign leads to an inversely conformal transformation. 
terpreted this equation geometrically as expressing zero mean curvature:

$$
\frac{1}{R_{1}}+\frac{1}{R_{2}}=0 .
$$

With the use of complex quantities, Monge (1784) integrated the partial differential equation of minimal surfaces, applying the parametric form of representation instead of the restricted form $z=f(x, y)$, and the formulas (1) of Weierstrass (1866) are essentially a transformation of those of Monge.

The problem of Plateau was taken up by Riemann [23], Weierstrass [24], and Schwarz [25], who confined themselves in the main to the case where the given contour is a skew polygon. They made the problem depend on a monodromy-group problem associated with a linear differential equation of the second order

$$
\frac{d^{2} \theta}{d w^{2}}+p \frac{d \theta}{d w}+q \theta=0,
$$

whose coefficients $p$ and $q$ are rational functions of $w$; but they did not succeed in obtaining a complete solution even for the polygonal case.

S. Bernstein (1910) [21] and A. Haar (1927) [20] did important work on the problem, using the restricted representation $z=f(x, y)$ of the surface. Bernstein took for basis the elliptic partial differential equation (4), and considered the Plateau problem as a generalized Dirichlet problem with the equation (4) taking the place of Laplace's equation $r+t=0$. Haar used the direct methods of the calculus of variations introduced by Hilbert. Both writers assumed the given contour to have a convex projection on the $x y$-plane.

The case of a contour of general shape has been considered only in recent years. R. Garnier (1928) [19] followed the classic methods of Riemann and Weierstrass, and concluded the existence of a minimal surface bounded by any given contour of (piece-wise) bounded curvature. Besides the general difficulty of following Garnier's developments to this conclusion, it is to be remarked that his work is based on that of Weierstrass, who assumes explicitly that the contour has no knots. $\dagger$ It is

$\dagger$ Weierstrass, Werke, vol. 3, p. 220, last line. 
therefore hard to see how Garnier frees himself of this restriction.

After the publication of the abstracts of the present author's papers in this Bulletin [1-10], T. Radó obtained by other methods results, which, as far as the Plateau problem is concerned, were not as general as those already found by the present author. Radó used the conformal mapping of polyhedral surfaces approximating to the required minimal surface, and restricted the contour first to be rectifiable [17], and then [18] to be capable of bounding a simply-connected surface of finite area.

The author, in collaboration with P. Franklin [16], has constructed an example of a step-polygon of a denumerable infinity of sides, every surface bounded by which has infinite area. Recently, I have constructed even simpler examples of Jordan space curves which bound no orientable surface of finite area $\dagger$, for instance, the spiral defined in spherical coordinates by the equations $r=\cos \phi, \theta=\tan ^{5} \phi$. The simplicity of these examples shows that one must be careful not to overestimate the generality of a contour that spans a finite area.

The method of solution here explained was developed by the author in the years 1926-1929. Besides the abstracts in this Bulletin, the essential features of the work were widely publicized by the author's lectures in mathematical seminars at Paris, Göttingen, and Hamburg during the year 1929. A comprehensive and didactic presentation was published in the Transactions of this Society in January, 1931, [11], followed by papers extending the theory to two contours [12] and to onesided minimal surfaces [13].

The advantages peculiar to the present treatment may be recapitulated as follows:

(i) The contour is subject to no restrictions whatever, except that it is any Jordan curve. The number of dimensions $n$ of the containing euclidean space is indifferent.

(ii) The conformal mapping theorem of Riemann for any Jordan region is included as the special case $n=2$.

(iii) The conformal map is derived from a topological correspondence between the boundaries, so that the theorem of Osgood and Carathéodory falls out as an immediate corollary. 2,4 .

$\dagger$ Proceedings of the National Academy of Sciences, vol. 19 (1933), Nos. 
3. The Weierstrass Proof of Attainment of a Minimum. The point and specific difficulty in the problem of Plateau is to prove that a certain minimum is attained, a minimum of area. The first to give a rigorous proof of the attainment of a minimum was Weierstrass, when he showed that every continuous function of a real variable on a closed interval attains its maximum and its minimum values.

The definition of a continuous function $f(x)$ is that if $x_{1}$, $x_{2}, \cdots, x_{n}, \cdots$ is any infinite sequence of points converging to a point $x^{*}$, so that $\lim _{n \rightarrow \infty} x_{n}=x^{*}$, then $\lim _{n \rightarrow \infty} f\left(x_{n}\right)=f\left(x^{*}\right)$.

The property of continuity may be divided into two properties, lower and upper semi-continuity. A function is lower semicontinuous if when

$$
\lim _{n \rightarrow \infty} x_{n}=x^{*}, \text { and } \lim _{n \rightarrow \infty} f\left(x_{n}\right)=L
$$

then

$$
f\left(x^{*}\right) \leqq L
$$

Similarly, a $\geqq$ sign in the last relation gives the definition of upper semi-continuous.

The theorem that a continuous function on a closed interval attains its extreme values may be divided into two parts: a lower semi-continuous function attains its minimum; an upper semi-continuous function attains its maximum. We need consider only the first of these, since the two are evidently equivalent by the transformation of $f(x)$ into $-f(x)$. Let us examine the simple details of its proof.

Let $m$ be the lower bound of the function $f(x)$ in the interval $(a b)$. The existence of $m$ as a finite quantity is implied by the lower semi-continuity of $f(x), \dagger$ but often, as in the present application, it is known a priori that $m$ exists and is finite, for instance, when $f(x)$ is an essentially positive quantity. Then, by definition of lower bound, we can always construct a "minimizing sequence"

$$
x_{1}, x_{2}, \cdots, x_{n}, \cdots,
$$

$\dagger$ This results from the present proof that $m$, finite or $-\infty$, is attained, that is, $m$ is one of the values of the function; and every value of $f(x)$ is supposed finite. 
such that

$$
f\left(x_{1}\right), f\left(x_{2}\right), \cdots, f\left(x_{n}\right), \cdots \rightarrow m .
$$

It is to be noted that it is only the functional values which tend to a limit; the argument values need not do so. Now we can apply the theorem of Bolzano-Weierstrass to the effect that the infinite sequence of points (7) on the finite closed interval $(a b)$ must contain a convergent subsequence

$$
x_{\perp}^{\prime}, x_{2}^{\prime}, \cdots, x_{n}^{\prime}, \cdots \rightarrow x^{*} .
$$

This property of any infinite set of points of the interval $(a b)$ is expressed by saying that this interval is a compact set of points. Of course, the limit point $x^{*}$ belongs to $(a b)$, since $(a b)$ is also a closed set. Together with (9), we have evidently

$$
f\left(x_{1}^{\prime}\right), f\left(x_{2}^{\prime}\right), \cdots, f\left(x_{n}^{\prime}\right), \cdots \rightarrow m,
$$

since (10) is a subsequence of (8); therefore, by the definition (6) of lower semi-continuity, it follows that $f\left(x^{*}\right) \leqq m$. But $f\left(x^{*}\right)<m$ is impossible, by definition of lower bound; consequently $f\left(x^{*}\right)=m$, which was to be proved; the minimum of $f(x)$ is attained at $x^{*}$. Such is the simple Weierstrass proof for the attainment of a minimum. It will be observed that it requires only the following two conditions:

(i) The argument range $R$ is compact and closed; that is, every infinite sequence of elements of $R$ contains a subsequence converging to a limit, which is also an element of $R$.

(ii) The function $f$ is lower semi-continuous.

A very general point of view as to functions on abstract sets of elements of any nature was developed by M. Fréchet [29] in his thesis, and the proof of attainment of a minimum was given by him as a consequence solely of the postulates (i) and (ii).

The present writer's contribution to the problem of Plateau is essentially this: by means of the new functional $A(g)$, to have given the problem an analytic formulation to which the Weierstrass-Fréchet proof for attainment of a minimum applies immediately. No treatment theretofore proposed does this.

That the functional $A(g)$ also unites the Plateau and Riemann problems of minimal surfaces and conformal mapping, is an additional mark of its appositeness and importance, since that, too, was not accomplished by any previous point of view. 
4. Comparison of Three Classic Problems. The classic analytic setting of the problem of Plateau was as a least area problem, the argument range consisting of all surfaces $[\Sigma]$ bounded by the given contour $\Gamma$, and the functional to be minimized being $S(\Sigma)$, the area of $\Sigma$. But to this formulation the WeierstrassFréchet reasoning does not apply because the range $[\Sigma]$ is not compact.

It will be instructive for purposes of comparison to consider also the Dirichlet problem. This is the problem of the existence of a function $\phi(x, y)$, taking prescribed values on the boundary of a given plane region and obeying in the interior of the region Laplace's equation $\partial^{2} \phi / \partial x^{2}+\partial^{2} \phi / \partial y^{2}=0$. This equation expresses the vanishing of the first variation of the integral

$$
D(\phi)=\iint\left[\left(\frac{\partial \phi}{\partial x}\right)^{2}+\left(\frac{\partial \phi}{\partial y}\right)^{2}\right] d x d y
$$

taken over the region, so that the problem is equivalent to the minimizing of $D(\phi)$ in the totality of functions of class $C^{\prime \prime} \dagger$ taking the prescribed boundary values.

The conformal mapping of a region $R$ on the interior of a circle depends on the solution of a Dirichlet problem for $R$. Let $G\left(x, y ; x_{0}, y_{0}\right)$ denote the Green's function for $R$. By definition, we have

$$
G\left(x, y ; x_{0}, y_{0}\right)=g\left(x, y ; x_{0}, y_{0}\right)+\log r,
$$

where $g$ is regular harmonic in $R, r^{2}=\left(x-x_{0}\right)^{2}+\left(y-y_{0}\right)^{2},\left(x_{0}, y_{0}\right)$ denotes an arbitrary point of $R$, and $G$ is required to reduce to zero on the boundary of $R$. This implies that $g\left(x, y ; x_{0}, y_{0}\right)$ $=-\log r$ on the boundary, so that the determination of the Green's function for $R$ consists of the solution of a certain Dirichlet problem. The Green's function having been found, the conformal mapping function can be written immediately as $e^{G+i H}$, where $H$ is the conjugate harmonic function to $G$.

Riemann believed he could assert the existence of $G$, or the solution of the associated Dirichlet problem, by the following argument: $D(\phi)$ is always a positive number; let $\phi^{*}$ be the function with the assigned boundary values for which $D(\phi)$ is least; then $\phi^{*}$ obeys Laplace's equation. Weierstrass pointed out the

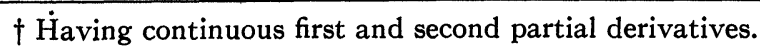


gap in this reasoning, which consists in the assumption involved in the italicized statement that there exists a function $\phi^{*}$ for which the minimum of $D(\phi)$ is attained. The cogency of the Weierstrass objection rests on the fact that the range of the argument $[\phi]$, consisting of all functions of class $C^{\prime \prime}$ in $R$ and with prescribed boundary values, is not compact. We can start by forming a minimizing sequence $\phi_{1}, \phi_{2}, \cdots, \phi_{n}, \cdots$, with

$$
D\left(\phi_{1}\right), D\left(\phi_{2}\right), \cdots, D\left(\phi_{n}\right), \cdots \rightarrow \min D(\phi),
$$

but nothing guarantees that this minimizing sequence contains a subsequence converging to a function of class $C^{\prime \prime}$ (or even converging at all). If this difficulty could be passed, the lower semi-continuity of $D(\phi)$ could then be used to prove the attainment of the minimum.

The typical and essential feature of the author's work on the Plateau problem is the introduction of a lower semi-continuous functional $A(g)$ whose argument range is compact. This functional is

$$
A(g)=\frac{1}{4 \pi} \int_{C} \int_{C} \frac{\sum_{i=1}^{n}\left[g_{i}(\theta)-g_{i}(\phi)\right]^{2}}{4 \sin ^{2} \frac{\theta-\phi}{2}} d \theta d \phi,
$$

whose argument $g$ is an arbitrary parametric representation $x_{i}=g_{i}(\theta)$ of the given contour $\Gamma$ as topological image of the unit circumference $C$.

The positive number thus associated with any topological correspondence between $\Gamma$ and $C$ may be interpreted as proportional to the mean square of the ratio of a chord of $\Gamma$ to the corresponding chord of $C$. The topological transformation $g^{*}$ of $C$ into $\Gamma$ which minimizes $A(g)$ and solves the Plateau problem may accordingly be regarded as the one which, on the average, brings the points of $C$ closest together [33].

The connection of the functional $A(g)$ with the Plateau problem is that if

$$
x_{i}=\Re F_{i}(w)
$$

denote the harmonic functions in the interior of the unit circle determined by the boundary values $g_{i}(\theta)$, which are given by 
Poisson's integral

$$
F_{i}(w)=\frac{1}{2 \pi} \int_{C} g_{i}(\theta) \frac{e^{i \theta}+w}{e^{i \theta}-w} d \theta,
$$

then the vanishing of the first variation of $A(g)$ expresses that $\sum_{i=1}^{n} F_{i}^{\prime 2}(w)=0$, which is the condition for a minimal surface.

The integral which expresses $A(g)$ is improper, on account of the singularity when $\theta=\phi$; but since the integrand is positive, $A(g)$ always has a definite value, finite and positive or $+\infty$. In the main body of the work we assume that $A(g)$ is not identically equal to $+\infty$; or, with the definition $m(\Gamma)=\min A(g)$, that $m(\Gamma)$ is finite. A sufficient condition for this is that $\Gamma$ be rectifiable. A more concrete interpretation of $m(\Gamma)$ is as the minimum area of all simply-connected surfaces bounded by $\Gamma . \dagger$ Afterwards, by an easy limit process, the existence proof is extended to the case $m(\Gamma)=+\infty$ of an arbitrary Jordan contour.

The following is a summary of the relationships between the three problems we have been comparing.

A. Dirichlet. (a) The argument range [ $\phi]$ consists of all functions of class $C^{\prime \prime}$ in a given region $R$ with assigned values on the boundary $\Gamma$ of $R$.

(b) The functional is

$$
D(\phi)=\iint_{R}\left[\left(\frac{\partial \phi}{\partial x}\right)^{2}+\left(\frac{\partial \phi}{\partial y}\right)^{2}\right] d x d y .
$$

(c) $D(\phi)$ is lower semi-continuous (positive integrand).

(d) $[\phi]$ is not compact.

(e) The Weierstrass-Fréchet reasoning cannot be applied to prove that $\min D(\phi)$ is attained.

(f) $\delta D(\phi)=0$ is Laplace's equation.

B. Least Area. (a) The argument range [ $\Sigma]$ consists of all surfaces of class $C^{\prime \prime}$ bounded by a given contour $\Gamma$.

(b) The functional is the area of $\Sigma$ :

$$
S(\Sigma)=\iint\left(E G-F^{2}\right)^{1 / 2} d u d v .
$$

$\dagger$ With this interpretation, $m(\Gamma)$ was first defined by Lebesgue [22 ], p. 304. 
(c) $S(\Sigma)$ is lower semi-continuous (positive integrand).

(d) $[\Sigma]$ is not compact.

(e) The Weierstrass-Fréchet reasoning cannot be applied to prove that $\min S(\Sigma)$ is attained.

(f) $\delta S(\Sigma)=0$ is $1 / R_{1}+1 / R_{2}=0$, which expresses the vanishing of the mean curvature of $\Sigma$.

C. Plateau-Riemann. (a) The argument range $[g]$ consists of all parametric representations of the given contour $\Gamma$, or all topological correspondences between $\Gamma$ and the unit circumference $C$.

(b) The functional is

$$
A(g)=\frac{1}{4 \pi} \int_{C} \int_{C} \frac{\sum_{i=1}^{n}\left[g_{i}(\theta)-g_{i}(\phi)\right]^{2}}{4 \sin ^{2}[(\theta-\phi) / 2]} d \theta d \phi .
$$

(c) $A(g)$ is lower semi-continuous (positive integrand).

(d) $[g]$ is compact.

(e) Therefore, by the Weierstrass-Fréchet reasoning, the minimum $A(g)$ is attained.

(f) $\delta A(g)=0$ is $\sum_{i=1}^{n} F_{i}^{\prime 2}(w)=0$, which expresses that the harmonic surface $x_{i}=\Re F_{i}(w)$ determined by the boundary values $x_{i}=g_{i}(\theta)$ is minimal and in conformal representation on the interior of the unit circle. For $n=2$, this gives the Riemann mapping theorem, together with the theorem of Osgood and Carathéodory.

5. Solution of the Problem of Plateau. OutLine. We shall first outline the successive steps in the solution, and then give the details of proof. An arbitrary parametric representation of $\Gamma$ can be defined by writing some fixed initial parameter $t$, which may be supposed to vary from 0 to $2 \pi$, as a monotonic increasing continuous function of $\theta$, the polar angle on the unit circle: $t=\mu(\theta)$. Since all these functions $\mu$ have the same total variation $2 \pi$, their set is compact, according to a theorem of Helly. $\dagger$ To obtain closure of the set, we must however introduce improper

$\dagger$ An infinite set of functions is compact if the functions are uniformly bounded and of uniformly bounded variation. As stated in geometric form by Fréchet [29], an infinite set of curves all contained in a finite region and of uniformly bounded lengths is compact. This theorem goes back to Hilbert, in his work on the Dirichlet principle, Jahresbericht der Deutschen Mathematiker-Vereinigung, vol. 8 (1899), p. 184. 
representations $g$, corresponding to functions $\mu$ whose graph contains line-segments parallel to the $t$ or $\theta$ axis. These cause a partial arc of $\Gamma$ to correspond to a point of $C$ (first kind), or a partial arc of $C$ to a point of $\Gamma$ (second kind); finally, there is the extreme case of a representation causing all of $\Gamma$ to correspond to a single point of $C$ and all of $C$ to a single point of $\Gamma$ (degenerate representation). Graphically, this is represented by a line segment of length $2 \pi$ along the $\theta$ axis joined to a line segment of length $2 \pi$ parallel to the $t$ axis, and must be included for closure of the set since evidently it can be obtained as a limit of proper monotonic graphs.

It will follow easily from the positive nature of the integrand of $A(g)$ that this functional is lower semi-continuous; hence, by the Weierstrass-Fréchet reasoning, the minimum of $A(g)$ is attained for a certain representation $g^{*}$.

The surface $x_{i}=\Re F_{i}(w)$ determined by $g^{*}$ according to the formula (13) obeys the condition $\sum_{i=1}^{n} F_{i}^{\prime 2}(w)=0$ characteristic of a minimal surface, for this condition, as will be proved, expresses that $\delta A(g)=0$ for $g=g^{*}$.

It remains to exclude the possibility that $g^{*}$ be improper or degenerate. The representation $g^{*}$ cannot be improper of the first kind, for it will be shown that then $A(g)=+\infty$, whereas, by assumption, $A(g)$ sometimes takes finite values. The case $g^{*}$ improper of the second kind will be ruled out with the help of the condition $\sum_{i=1}^{n} F_{i}^{\prime 2}(w)=0$.

The possibility that $g^{*}$ is degenerate will be prevented by the observation that $A(g)$ is invariant under linear fractional transformation of the unit circumference into itself :

$$
\tan \frac{\theta^{\prime}}{2}=\frac{a \tan (\theta / 2)+b}{c \tan (\theta / 2)+d}
$$

involving three arbitrary constants $a: b: c: d$. Therefore no generality is lost if we fix three distinct points $P_{1}, P_{2}, P_{3}$ of $C$ and three distinct points $Q_{1}, Q_{2}, Q_{3}$ of $\Gamma$, and consider only those topological correspondences between $C$ and $\Gamma$ which cause these points to correspond respectively. Then at most the sum of two of the arcs $P_{1} P_{2}, P_{2} P_{3}, P_{3} P_{1}$ can correspond to a point of $\Gamma$, and the sum of two of the $\operatorname{arcs} Q_{1} Q_{2}, Q_{2} Q_{3}, Q_{3} Q_{1}$ to a point of $C$. The degenerate representations are thus excluded. 
The minimizing representation $g^{*}$ is therefore proper; consequently the minimal surface determined by it is bounded by $\Gamma$, for Poisson's integral attaches continuously to its boundary values at every point $\theta$ where these values are continuous, and a proper representation is everywhere continuous.

This is the outline of the solution of the Plateau problem for all contours with finite $m(\Gamma)$. The case $m(\Gamma)=+\infty$ will then be dealt with by means of a simple limit process.

We proceed to develop the details. First, we write an expression for $A(g)$ in terms of complex variables:

$$
A(g)=\frac{1}{4 \pi} \int_{C} \int_{C} \sum_{i=1}^{n}\left[g_{i}(z)-g_{i}(\zeta)\right]^{2} \frac{d z d \zeta}{(z-\zeta)^{2}},
$$

where

$$
z=e^{i \theta}, \zeta=e^{i \phi}
$$

vary over the unit circumference $C$, regarded as contained in the complex plane. The identity of (14) with (11) is immediate, by the substitution (15). Another way of writing (14) is

$$
A(g)=\frac{1}{4 \pi} \int_{C} \int_{C} \sum_{i=1}^{n}\left[g_{i}(z)-g_{i}(\zeta)\right]^{2} \cdot(z, z+d z, \zeta, \zeta+d \zeta)
$$

where the parenthesis denotes an anharmonic ratio. From this it is evident that, although complex quantities enter into the element of integration, its value is real and positive, since $z$, $z+d z, \zeta, \zeta+d \zeta$ are concyclic points and the first pair is not separated by the second. This positive real character is, of course, also known through the identity of (14) with (11).

It follows immediately from the form (16) that $A(g)$ is invariant under any linear fractional transformation

$$
z^{\prime}=\frac{\alpha z+\beta}{\gamma z+\delta},
$$

where $\alpha, \beta, \gamma, \delta$ are any complex quantities such that $\alpha \delta-\beta \gamma$ $\neq 0$. This is the invariance property of $A(g)$ used in the preceding outline to avoid the degenerate representations. The transformation (17) also shows that any circle $C$ in the complex plane may be used instead of the unit circle on which to represent $\Gamma$ parametrically, $A(g)$ being defined by (14) or (16). 
Lower Semi-Continuity. That the functional $A(g)$ is lower semi-continuous is to be seen as follows. We have to define $A(g)$ as an improper integral by the formula $A(g)=\lim _{\epsilon \rightarrow 0}$ $A_{\epsilon}(g)$, where $A_{\epsilon}(g)$ is defined by (14) with the variables of integration restricted by $|z-\zeta| \geqq \epsilon$. The functional $A_{\epsilon}(g)$ is, for every fixed $\epsilon>0$, continuous, which means that if $g$ is a variable representation of $\Gamma$ which approaches a certain fixed representation as limit, then $\lim A_{\epsilon}(g)=A_{\epsilon}(\lim g)$. This results from a theorem of Lebesgue, according to which one may pass to the limit under the sign of integration provided the integrand remains uniformly bounded; and a uniform upper bound for the absolute value of the integrand of $A_{\epsilon}(g)$ is $d^{2} / \epsilon^{2}$, where $d$ is the diameter (greatest chord) of $\Gamma$.

The approach of $A_{\epsilon}(g)$ to $A(g)$ as $\epsilon \rightarrow 0$ is in a monotonic increasing way, since diminishing $\epsilon$ adds positive elements to the integral. It is easy to prove the following lemma. $\dagger$

A functional is lower semi-continuous if it can be represented as the limit of a continuous functional which tends to it in increasing.

Hence $A(g)$ is lower semi-continuous. This, with the compactness and closure of the set $[g]$, proves the existence of $g^{*}$ for which the minimum of $A(g)$ is attained.

EXCLUSION OF IMPROPER REPRESENTATIONS OF THE First KIND. To exclude the possibility that $g^{*}$ is improper of the first kind, let $l>0$ be the length $\ddagger$ of the chord of the arc of $\Gamma$ which corresponds in the improper representation to the point $P$ of $C$; then if $z$ and $\zeta$ are on opposite sides of $P$ and approach to it, the integrand of $A(g)$ has asymptotically the form $\left[l^{2} /(z-\zeta)^{2}\right] d z d \zeta$, of which the indefinite integral is $l^{2} \log (z-\zeta)$, and this becomes infinite for $z=\zeta$, giving the result $A(g)=+\infty$.

We now know that $g^{*}$ is proper or, at the worst, improper in such a manner that it remains constantly at a fixed point of $\Gamma$ while $z$ describes a partial arc of $C$. Accordingly, $g^{*}$ is, in any event, continuous. We can then form, with the boundary values $g=g^{*}$, Poisson's integral:

$$
x_{i}=\Re F_{i}(w), \quad F_{i}(w)=\frac{1}{2 \pi i} \int_{C} g_{i}(z) \frac{z+w}{z-w} \frac{d z}{z},
$$

$\dagger[11]$, p. 282.

$\ddagger$ In the condition $l>0$, we are using the assumption that the contour has no double points; the end-points of any partial arc of $\Gamma$ are distinct. 
which will define a harmonic surface bounded by $\Gamma$.

Vanishing of the First Variation of $A(g)$. The point now is that the vanishing of the first variation of $A(g)$ for $g=g^{*}$,

$$
\delta A(g)=0,
$$

expresses exactly the condition

$$
\sum_{i=1}^{n} F_{i}^{\prime 2}(w)=0
$$

which characterizes as a minimal surface the one defined by the formulas (18). After (20) has been established, it will be easy to get rid of the remaining possibility that $g^{*}$ be improper of the second kind.

In proceeding to the proofs, we first derive from (18), by a permissible differentiation under the integral sign, the formula

$$
F_{i}^{\prime}(w)=\frac{1}{\pi i} \int_{C} g_{i}(z) \frac{d z}{(z-w)^{2}},
$$

and therefrom

$$
\sum_{i=1}^{n} F_{i}^{\prime 2}(w)=-\frac{1}{\pi^{2}} \int_{C} \int_{C} \sum_{i=1}^{n} g_{i}(z) g_{i}(\zeta) \frac{d z d \zeta}{(z-w)^{2}(\zeta-w)^{2}},
$$

whence

(22) $\sum_{i=1}^{n} F_{i}^{\prime 2}(w)=\frac{1}{2 \pi^{2}} \int_{C} \int_{C} \sum_{i=1}^{n}\left[g_{i}(z)-g_{i}(\zeta)\right]^{2} \frac{d z d \zeta}{(z-w)^{2}(\zeta-w)^{2}}$,

where the terms $g_{i}(z)^{2}$ and $g_{i}(\zeta)^{2}$ in the expansion of the bracket contribute zero, since

$$
\int_{C} \frac{d \zeta}{(\zeta-w)^{2}}=0, \quad \int_{C} \frac{d z}{(z-w)^{2}}=0
$$

Next we observe that (using the subscript zero to denote the conjugate complex quantity)

$$
\frac{1}{z(z-w)} \text { and } \frac{1}{z_{0}\left(z_{0}-w_{0}\right)}
$$


are conjugate complex; consequently

$$
\lambda\left\{\frac{1}{z(z-w)}-\frac{1}{z_{0}\left(z_{0}-w_{0}\right)}\right\} \text { and } i \lambda\left\{\frac{1}{z(z-w)}+\frac{1}{z_{0}\left(z_{0}-w_{0}\right)}\right\}
$$

are pure imaginary, where $\lambda$ denotes a real parameter and $w$ any fixed point interior to the unit circle. Hence the transformations

$$
\begin{aligned}
& z^{\prime}=z \exp \lambda\left\{\frac{1}{z(z-w)}-\frac{1}{z_{0}\left(z_{0}-w_{0}\right)}\right\}, \\
& z^{\prime}=z \exp i \lambda\left\{\frac{1}{z(z-w)}+\frac{1}{z_{0}\left(z_{0}-w_{0}\right)}\right\}
\end{aligned}
$$

convert points of $C$ into points of $C$, since they leave $|z|$ invariant. For $\lambda=0$ these transformations reduce to $z^{\prime}=z$, or on $C, \theta^{\prime}=\theta$, for which $d \theta^{\prime} / d \theta=1$. Therefore, for $\lambda$ small enough absolutely, $d \theta^{\prime} / d \theta$ certainly remains positive since it is nearly equal to one; consequently the transformations (23) and (24) are, for $|\lambda|$ sufficiently small, monotonic continuous transformations of the unit circumference into itself. Accordingly, if we apply (23), together with the same transformation $\zeta \rightarrow \zeta^{\prime}$, to $A\left(g^{*}\right)$, we get a function $A_{1}(\lambda)$ with a relative minimum at $\lambda=0$.

The transformation (23) may be expanded in a convergent power series in $\lambda$ :

$$
z^{\prime}=z+\lambda\left\{\frac{1}{z-w}-\frac{z}{z_{0}\left(z_{0}-w_{0}\right)}\right\}+\cdots
$$

from which results a convergent power series for the differential :

$$
\frac{d z^{\prime} d \zeta^{\prime}}{\left(z^{\prime}-\zeta^{\prime}\right)^{2}}=\frac{d z d \zeta}{(z-\zeta)^{2}}+\lambda\{\cdots\}+\cdots
$$

Multiplying this by

$$
\sum_{i=1}^{n}\left[{ }^{\prime} g_{i}\left(z^{\prime}\right)-{ }^{\prime} g_{i}\left(\zeta^{\prime}\right)\right]^{2}=\sum_{i=1}^{n}\left[g_{i}(z)-g_{i}(\zeta)\right]^{2},
$$


and integrating term by term, $\uparrow$ we get a power series expansion for $A_{1}(\lambda)$ of the form $A_{1}(\lambda)=A\left(g^{*}\right)+\lambda V_{1}+\cdots$. Because of the minimum of $A_{1}(\lambda)$ at $\lambda=0$, we deduce

$$
V_{1}=0 \text {. }
$$

Similarly, applying the transformation (24) in expanded form

$$
z^{\prime}=z+i \lambda\left\{\frac{1}{z-w}+\frac{z}{z_{0}\left(z_{0}-w_{0}\right)}\right\}+\cdots,
$$

we get $A_{2}(\lambda)=A\left(g^{*}\right)+i \lambda V_{2}+\cdots$, and hence

$$
V_{2}=0 \text {. }
$$

Now it is evident, as a formal matter, that we would get the same first power coefficients, or variations $V_{1}, V_{2}$, if we broke off (25) and (27) after the terms written. It is clear also that if we use any linear combination of the coefficients in (25) and (27), such as one-half the sum:

$$
z^{\prime}=z+\frac{\lambda}{z-w},
$$

and apply this transformation to $A\left(g^{*}\right)$, thus getting an expansion

$$
A\left(g^{*}\right)+\lambda V_{3}+\cdots,
$$

then $V_{3}$ is the same linear combination of $V_{1}$ and $V_{2}$ :

$$
V_{3}=\frac{1}{2}\left(V_{1}+V_{2}\right)
$$

consequently, by (26) and (28),

$$
V_{3}=0 .
$$

We therefore apply the simple variation (29) to $A\left(g^{*}\right)$ and calculate $V_{3}$. The following are the computations, all terms beyond the first power of $\lambda$ being neglected:

$\dagger$ The validity of this operation is easily established by classic convergence theorems (Cauchy appraisal theorem, Weierstrass double-series theorem); see [1], $\$ 13,14$. 


$$
\begin{gathered}
z^{\prime}=z+\frac{\lambda}{z-w}, \quad \zeta^{\prime}=\zeta+\frac{\lambda}{\zeta-w}, \\
d z^{\prime}=\left\{1-\frac{\lambda}{(z-w)^{2}}\right\} d z, \quad d \zeta^{\prime}=\left\{1-\frac{\lambda}{(\zeta-w)^{2}}\right\} d \zeta \\
d z^{\prime} d \zeta^{\prime}=\left\{1-\frac{\lambda}{(z-w)^{2}}-\frac{\lambda}{(\zeta-w)^{2}}\right\} d z d \zeta, \\
z^{\prime}-\zeta^{\prime}=(z-\zeta)\left\{1-\frac{\lambda}{(z-w)(\zeta-w)}\right\}, \\
\frac{d z^{\prime} d \zeta^{\prime}}{\left(z^{\prime}-\zeta^{\prime}\right)^{2}}=\frac{d z d \zeta}{(z-\zeta)^{2}}\left\{1-\lambda\left[\frac{1}{(z-w)}-\frac{1}{(\zeta-w)}\right]^{2}\right\}
\end{gathered}
$$

The coefficient of $\lambda$ is

$$
\left(\frac{1}{z-w}-\frac{1}{\zeta-w}\right)^{2}=\frac{(z-\zeta)^{2}}{(z-w)^{2}(\zeta-w)^{2}}
$$

hence

$$
\frac{d z^{\prime} d \zeta^{\prime}}{\left(z^{\prime}-\zeta^{\prime}\right)^{2}}=\frac{d z d \zeta}{(z-\zeta)^{2}}-\lambda \frac{d z d \zeta}{(z-w)^{2}(\zeta-w)^{2}} .
$$

Multiplying this by

$$
\sum_{i=1}^{n}\left[g_{i}^{*}\left(z^{\prime}\right)-{ }^{\prime} g_{i}^{*}\left(\zeta^{\prime}\right)\right]^{2}=\sum_{i=1}^{n}\left[g_{i}^{*}(z)-g_{i}^{*}(\zeta)\right]^{2},
$$

and integrating, we get a power series expansion in $\lambda$, beginning

$$
\begin{aligned}
& \frac{1}{4 \pi} \int_{C} \int_{C} \sum_{i=1}^{n}\left[{ }^{\prime} g_{i}^{*}\left(z^{\prime}\right)-{ }^{\prime} g_{i}^{*}\left(\zeta^{\prime}\right)\right]^{2} \frac{d z^{\prime} d \zeta^{\prime}}{\left(z^{\prime}-\zeta^{\prime}\right)^{2}} \\
& \quad=A\left(g^{*}\right)-\lambda \cdot \frac{1}{4 \pi} \int_{C} \int_{C} \sum_{i=1}^{n}\left[g_{i}^{*}(z)-g_{i}^{*}(\zeta)\right]^{2} \frac{d z d \zeta}{(z-w)^{2}(\zeta-w)^{2}} .
\end{aligned}
$$

Comparing with (30), we have therefore, by (31),

$$
\int_{C} \int_{C} \sum_{i=1}^{n}\left[g_{i}{ }^{*}(z)-g_{i}{ }^{*}(\zeta)\right]^{2} \frac{d z d \zeta}{(z-w)^{2}(\zeta-w)^{2}}=0 .
$$

By (22), this is the same as 


$$
\sum_{i=1}^{n} F_{i}^{\prime 2}(w)=0
$$

which proves that the surface $x_{i}=\Re F_{i}(w)$ determined by $g^{*}$ is minimal.

EXClusion of Improper Representations of the Second KIND. To show now that $g^{*}$ cannot be constant on a partial arc of $C$, we shall first introduce into (33) the factor $w^{2}$, by writing $\sum_{i=1}^{n} w^{2} F_{i}^{\prime 2}(w)=0$, and set $\dagger$

$$
w F_{i}^{\prime}(w)=\Re w F_{i}^{\prime}(w)+i \Im w F_{i}^{\prime}(w) ;
$$

and we shall then take the real part of the resulting equation, getting

$$
\sum_{i=1}^{n}\left\{\Re w F_{i}^{\prime}(w)\right\}^{2}=\sum_{i=1}^{n}\left\{\Im w F_{i}^{\prime}(w)\right\}^{2} .
$$

Multiplying (21) by $w$ and then expressing in terms of polar coordinates, we have $w=\rho e^{i \alpha}, z=e^{i \theta}$; and, separating out the imaginary part, we find

(35) $\Im w F_{i}^{\prime}(w)=-\frac{1}{2 \pi} \int_{C} \frac{2 \rho\left(1-\rho^{2}\right) \sin (\theta-\alpha)}{\left[1-2 \rho \cos (\theta-\alpha)+\rho^{2}\right]^{2}} g_{i}^{*}(\theta) d \theta$.

According to a result of Fatou [30], the limit of this harmonic function as $w$ approaches a boundary point $e^{i \theta}$ exists as a unique value and is equal to the derivative $d g^{*}(\theta) / d \theta$ at every point where this derivative exists and is continuous.

Since, by the hypothesis which we are trying todisprove, $g^{*}(\theta)$ is constant on a partial arc of $C$, it follows that $\Im w F_{i}^{\prime}(w)$ $=0$ on this arc, for $i=1,2, \cdots, n$. Hence, by $(34), \Re w F_{i}^{\prime}(w)=0$, also for $i=1,2, \cdots, n$, on the same arc. Consequently $w F_{i}^{\prime}(w)=0$ on the arc, for $i=1,2, \cdots, n$. This enables us to conclude that $w F_{i}{ }^{\prime}(w) \equiv 0,(i=1,2, \cdots, n)$, since, by Schwarzian symmetry, involving inversion in $C$, the arc can be made interior to a domain of regularity of the function.

Thus $F_{i}(w)$ would reduce to a constant, and consequently also $g_{i}{ }^{*}$, the boundary value of $\Re F_{i}(w)$. But we have assumed that $g^{*}$ is constant only on a partial arc of $C$, the possibility of

$\dagger$ Of course, there should be no confusion of $i$, the index that runs from 1 to $n$, with $i$, the square root of -1 . 
a degenerate representation having been previously ruled out by the condition of three distinct fixed points on $\Gamma$ and $C$.

The contradiction thus exhibited completes the reasoning. The minimizing representation $g^{*}$ of $A(g)$ is proper, and the minimal surface determined by it is therefore bounded by $\Gamma$. This solves the Plateau problem for any contour $\Gamma$ with finite $m(\Gamma)$.

Arbitrary Jordan Contour. To treat the case $m(\Gamma)=+\infty$, we represent the contour $\Gamma$ as limit of a sequence of contours $\bar{\Gamma}$ with finite $m(\bar{\Gamma})$, for instance, polygons. Let $x_{i}=\bar{f}_{i}(t)$ be the equations of $\bar{\Gamma}, x_{i}=f_{i}(t)$ of $\Gamma$; then $\bar{f}_{i}(t) \rightarrow f_{i}(t)$. Each contour $\bar{\Gamma}$ has a parameter $\theta$ solving the Plateau problem for $\bar{\Gamma}, \theta$ being derived from $t$ by a monotonic continuous transformation $t=\bar{\mu}(\theta)$. The sequence of monotonic transformations $\bar{\mu}$, by the compactness of the set of all such transformations, contains a convergent subsequence, with limit a proper or improper monotonic transformation $t=\mu(\theta)$. We thus obtain a certain representation of $\Gamma$ in the form

$$
x_{i}=g_{i}(\theta)=f_{i}(\mu(\theta))
$$

where $g_{i}(\theta)$ is the limit of a sequence of Plateau representations of $\bar{\Gamma}$,

$$
x_{i}=\bar{g}_{i}(\theta)=\bar{f}_{i}(\bar{\mu}(\theta)) \text {. }
$$

If $x_{i}=\Re F_{i}(w)$ and $\bar{x}_{i}=\Re \bar{F}_{i}(w)$ are formed respectively from $g, \bar{g}$ by the Poisson formula (18), then evidently, with $F^{\prime}(w)$, $\bar{F}^{\prime}(w)$ given by $(21)$, we have $\bar{F}_{i}^{\prime}(w) \rightarrow F_{i}^{\prime}(w)$, for the integrand remains uniformly bounded for any fixed value of $w$. Since during the limit process we have always $\sum_{i=1}^{n} \bar{F}_{i}^{\prime 2}(w)=0$, it follows that

$$
\sum_{i=1}^{n} F_{i}^{\prime 2}(w)=0
$$

It remains to prove that $x_{i}=g_{i}(\theta)$ is a proper representation of $\Gamma$. The condition (36) having been established, the reasoning gone through just previously, based on Fatou's theorem and Schwarzian symmetry, applies to rule out improper representations of the second kind. The possibility of a degenerate representation is excluded by restricting the monotonic transformations $\bar{\mu}$ to leave fixed three chosen distinct points of the unit 
circumference, as can be done without harm to generality by use of a linear fractional transformation of the unit circle into itself, this involving three arbitrary constants.

However, the possibility of an improper representation of the first kind cannot now be excluded by the argument given for the case of finite $m(\Gamma)$ : that then $A(g)=+\infty$, for here $A(g)$ is identically $+\infty$. To rule out this type of improper representation, we now show that it is inconsistent with (36).

In an improper representation of the first kind there is a point $P$ on the unit circumference where the vector function $g$ has unequal one-sided limits, the distinct points $\left(a_{i}\right)$ and $\left(b_{i}\right)$ of $\Gamma$. Without loss of generality we may rotate the unit circle till $P$ is at $w=1$.

We next invert the unit circle with respect to an orthogonal circle whose center $O$ is on the radius to $P$ prolonged. $\dagger$ This inversion, combined with a reflection in the real axis, gives a transformation

$$
w^{\prime}=\frac{\alpha w+\beta}{\gamma w+\delta},
$$

which converts the unit circle into itself, and converts the functions $F_{i}(w)$ into new ones which evidently still obey the relation (36).

If now $O$ is made to tend to $P$, it is seen that the transformation (37) tends to spread the values of $g$ just below $P$ over the whole upper semi-circumference and the values just above $P$ over the whole lower semi-circumference, so that $g_{i}$ tends to the function equal to $a_{i}$ on the upper semi-circumference and to $b_{i}$ on the lower.

Consequently, by formula (13), $F_{i}(w)$ tends to the function

$$
\begin{aligned}
G_{i}(w) & =\frac{1}{2 \pi} \int_{0}^{\pi} a_{i} \frac{e^{i \theta}+w}{e^{i \theta}-w} d \theta+\frac{1}{2 \pi} \int_{\pi}^{2 \pi} b_{i} \frac{e^{i \theta}+w}{e^{i \theta}-w} d \theta \\
& =\frac{a_{i}-b_{i}}{\pi i} \log i \frac{1+w}{1-w} .
\end{aligned}
$$

At the same time $F_{i}^{\prime}(w)$ tends to $G_{i}^{\prime}(w)$, and $\sum_{i=1}^{n} F_{i}^{\prime 2}(w)$ to $\sum_{i=1}^{n} G_{i}^{\prime 2}(w)$. But, as calculated from (38),

† See [11], p. 305. 


$$
\sum_{i=1}^{n} G_{i}^{\prime 2}(w)=-\frac{4}{\pi^{2}} \sum_{i=1}^{n}\left(a_{i}-b_{i}\right)^{2} \cdot \frac{1}{\left(1-w^{2}\right)^{2}},
$$

which is $\neq 0$, since the points $\left(a_{i}\right)$ and $\left(b_{i}\right)$ are distinct. $\dagger$ Thus we have contradiction with the fact that in the limit process in which $O \rightarrow P$ the condition $\sum_{i=1}^{n} F_{i}^{\prime 2}(w)=0$ is always obeyed.

LEAst AREa. Having taken the Weierstrass formulas as definition of minimal surface, we have not concerned ourselves in the preceding account with the least area property of the minimal surface $M$ whose existence has now been established. It is, however, a fact that $M$ has the minimum area of any simply-connected surface bounded by $\Gamma[11, \S 26 ; 15]$.

This statement is significant only in case the minimum area $m(\Gamma)$ is finite. But even when $m(\Gamma)=+\infty$, there is still a good sense in which the least area property is had by the surface $M$. Let $\Gamma^{\prime}$ denote any closed contour on $M$ having no point in common with the boundary $\Gamma$. Then the area enclosed by $\Gamma^{\prime}$ on $M$ is finite and an absolute minimum with respect to all simplyconnected surfaces bounded by $\Gamma^{\prime}[14]$. That is, the infinite part of the area of $M$ lies, so to say, altogether on its boundary. If $\Gamma_{\rho}$ denote the contour on $M$ corresponding to the circle of radius $\rho<1$ concentric with the unit circle, then as $\rho \rightarrow 1$ the area enclosed on $M$ by $\Gamma_{\rho}$ becomes infinite in at most the order of $(1-\rho)^{-3}$; in fact, if $A_{\rho}$ denote the area intercepted on $M$ by $\Gamma_{\rho}$, then, for all $\rho<1$, we have

$$
A_{\rho} \leqq \frac{3 \pi D^{2}}{(1-\rho)^{3}},
$$

where $D$ denotes the length of the diagonal of any rectangular parallelepiped containing the contour $\Gamma$.

6. Conformal Mapping. To get the Riemann mapping theorem for Jordan regions together with the Osgood-Carathéodory theorem concerning the behavior of the map on the boundaries is merely a matter of putting $n=2$ in the preceding work.

Let the plane Jordan curve $\Gamma$ bound a region $R$. Then, by the foregoing theory, there exists a topological correspondence between $\Gamma$ and the unit circumference $C$ that gives a parametric representation of $\Gamma$ of the form

$\dagger$ Here again, use is being made of the condition that the contour has no double points. 


$$
x_{1}=g_{1}^{*}(z), x_{2}=g_{2}^{*}(z),
$$

such that the functions

$$
\begin{aligned}
& F_{1}(w)=\frac{1}{2 \pi i} \int_{C} g_{1} *(z) \frac{z+w}{z-w} \frac{d z}{z}, \\
& F_{2}(w)=\frac{1}{2 \pi i} \int_{C} g_{2} *(z) \frac{z+w}{z-w} \frac{d z}{z}
\end{aligned}
$$

obey the condition

$$
F_{1}^{\prime 2}(w)+F_{2}^{\prime 2}(w)=0 \text {, or } F_{1}^{\prime}(w) \pm i F_{2}^{\prime}(w)=0 .
$$

We have seen in $\$ 1$ that this implies that the functions

$$
x_{1}=\Re F_{1}(w), \quad x_{2}=\Re F_{2}(w)
$$

are conjugate harmonic, or that

$$
W=x_{1}+i x_{2}=F(w)
$$

is a monogenic function of $w$. As such, it is therefore expressible in terms of its boundary values on $C$ in the form

$$
g^{*}(z)=g_{1}^{*}(z)+i g_{2}^{*}(z)
$$

by the Cauchy integral formula

$$
F(w)=\frac{1}{2 \pi i} \int_{C} \frac{g^{*}(z) d z}{z-w} .
$$

The equations (44) and (45) are merely combined forms of (43) and (42).

Because the transformation (44) converts the unit circumference in a one-to-one continuous way into $\Gamma$, it follows, by an important theorem of Darboux, $\dagger$ that it converts the interior of the unit circle into the interior $R$ of $\Gamma$ in a one-to-one manner and conformally, without singular points. The mapping theorem of Riemann is proved.

To obtain now the theorem of Osgood and Carathéodory requires only the simple observation that the formulas (42) and (43) which define the conformal map are equivalent to Poisson's integral, which has the well known property of attaching

† Osgood, Funktionentheorie, 1923, p. 377. 
continuously to its boundary values $g_{1}^{*}(z)$ and $g_{2}^{*}(z)$, if these are continuous, as they are.

For any contour $\Gamma$, it is easy to show that the minimum value of $A(g)$ is equal to the area of the minimal surface $M$ determined by $g^{*}$. Therefore, in the case of a plane Jordan curve bounding a region $R$,

$$
\min A(g)=\text { inner area of } R .
$$

The inner area means the upper bound of the areas of all polygons which, with their boundaries, are interior to $R . \dagger$ This remark made, we can now summarize the results of this section in the following proposition.

Let $\Gamma$ denote any plane Jordan curve bounding a region $R$, and let $Z=g(z)$ denote an arbitrary representation of $\Gamma$, locus of $Z$, as topological image of the unit circumference $C$, locus of $z$. The range of values of the functional

$$
A(g)=\frac{1}{4 \pi} \int_{C} \int_{C}|g(z)-g(\zeta)|^{2} \frac{d z d \zeta}{(z-\zeta)^{2}}
$$

when all representations $g$ are considered will consist exactly of all positive real numbers $\geqq$ the inner area of $R$. This minimum value is attained for a certain representation $Z=g^{*}(z)$ (determined up to linear fractional transformation of $C$ into itself). Then the transformation $w \rightarrow W$ defined by the integral formula of Cauchy,

$$
W=\frac{1}{2 \pi i} \int_{C} \frac{g^{*}(z) d z}{z-w}
$$

establishes a one-to-one conformal map of the interior of the unit circle on the region $R$, and this conformal map attaches continuously to the topological correspondence $g^{*}$ between $\Gamma$ and $C$.

7. Dirichlet Principle. The implications for the Dirichlet problem of the results just stated are apparent. The Dirichlet problem for any continuous distribution of assigned values on

$\dagger$ To be distinguished from the outer area, lower bound of the areas of all simply-connected polygons whose boundaries are exterior to $R$. The first example of a Jordan curve with inner area less than outer area, or where the curve itself has a positive area, was given by Osgood, Transactions of this Society, vol. 4 (1903), pp. 107-112. 
any Jordan curve is reduced to the same problem for the circle, and therefore solved immediately by Poisson's integral.

It will be observed that the proof of the Dirichlet principle thus given consists in introducing instead of the Dirichlet functional $D(\phi)$, against which the Weierstrass objection applies (the range of the argument $[\phi]$ is not compact), the functional $A(g)$ which, on the contrary, is amenable to immediate treatment according to the Weierstrass-Fréchet pattern.

\section{BIBLIOGRAPHY*}

1. J. Douglas, Reduction of the problem of Plateau to an integral equation, this Bulletin, vol. 33 (1927), p. 143.

2. J. Douglas, Reduction to integral equations of the problem of Plateau for the case of two contours, this Bulletin, vol. 33 (1927), p. 259.

3. J. Douglas, Reduction of the problem of Plateau to the minimization of a certain functional, this Bulletin, vol. 34 (1928), p. 405.

4. J. Douglas, Solution of the problem of Plateau, this Bulletin, vol. 35 (1929), p. 292.

5. J. Douglas, Various forms of the fundamental functional in the problem of Plateau and its relation to the area functional, this Bulletin, vol. 36 (1930), p. 49.

6. J. Douglas, $A$ general formulation of the problem of Plateau, this Bulletin, vol. 36 (1930), p. 50.

7. J. Douglas, Solution of the problem of Plateau for any rectifiable contour in n-dimensional euclidean space, this Bulletin, vol. 36 (1930), p. 189.

8. J. Douglas, Solution of the problem of Plateau when the contour is an arbitrary Jordan curve in n-dimensional euclidean space, I, this Bulletin, vol. 36 (1930), p. 189.

9. J. Douglas, Solution of the problem of Plateau when the contour is an arbitrary Jordan curve in n-dimensional euclidean space, II, this Bulletin, vol. 36 (1930), p. 190.

10. J. Douglas, The problem of Plateau and the theorem of Osgood-Carathéodory on the conformal mapping of Jordan regions, this Bulletin, vol. 36 (1930), p. 190.

11. J. Douglas, Solution of the problem of Plateau, Transactions of this Society, vol. 33 (1931), pp. 263-321.

12. J. Douglas, The problem of Plateau for two contours, Journal of Mathematics and Physics of the Massachusetts Institute of Technology, vol. 10 (1931), pp. 315-359; vol. 11 (1932), p. 212.

13. J. Douglas, One-sided minimal surfaces with a given boundary, Transactions of this Society, vol. 34 (1932), pp. 731-756.

14. J. Douglas, The least area property of the minimal surface determined by an arbitrary Jordan contour, Proceedings of the National Academy of Sciences, vol. 17 (1931), pp. 211-216.

* Nos. 1-10 listed below are abstracts of papers presented to this Society. 
15. J. Douglas, The mapping theorem of Koebe and the problem of Plateau, Journal of Mathematics and Physics of the Massachusetts Institute of Technology, vol. 10 (1931), pp. 106-130.

16. J. Douglas and P. Franklin, $A$ step-polygon of a denumerable infinity of sides which bounds no finite area, Proceedings of the National Academy of Sciences, vol. 19 (1933), pp. 188-191.

17. T. Rad6, On Plateau's problem, Annals of Mathematics, vol. 31 (1930), pp. $457-469$.

18. T. Rado, The problem of least area and the problem of Plateau, Mathematische Zeitschrift, vol. 32 (1930), pp. 762-796.

19. R. Garnier, Le problème de Plateau, Annales de l'École Normale Supérieure, vol. 45 (1928), pp. 53-144.

20. A. Haar, Ueber das Plateausche Problem, Mathematische Annalen, vol. 97 (1927), pp. 124-158.

21. S. Bernstein, Sur le problème de Dirichlet généralisé, Mathematische Annalen, vol. 69 (1910), pp. 82-136, esp. \$18.

22. H. Lebesgue, Intégrale, longueur, aire, Annali di Matematica, vol. 7 (1902), pp. 231-359.

23. B. Riemann, Werke, 1892, pp. 301-337, 445-454.

24. K. Weierstrass, Werke, 1903, vol. 3, pp. 39-52, 219-220, 221-238.

25. H. Schwarz, Gesammelte Mathematische Abhandlungen, 1890, vol. 1.

26. G. Darboux, Leçons sur la Théorie Générale des Surfaces, (2d ed.) 1914, pp. $490-601$.

27. J. Plateau, Statique Expérimentale et Théorique des Liquides, 1873.

28. J. L. Lagrange, Oeuvres, vol. 1, p. 335.

29. M. Fréchet, Sur quelques points du calcul fonctionnel, Rendiconti di Palermo, vol. 22 (1906), pp. 1-74.

30. P. Fatou, Séries trigonométriques et séries de Taylor, Acta Mathematica, vol. 30 (1906), pp. 347-348.

31. W. F. Osgood and E. H. Taylor, Conformal transformations on the boundaries of their regions of definition, Transactions of this Society, vol. 4 (1913), pp. 277-298.

32. C. Carathéodory, Ueber die gegenseitige Beziehungen der Ränder bei der konformen Abbildung des Innern einer Jordanschen Kurve auf einen Kreis, Mathematische Annalen, vol. 73 (1913), pp. 305-320.

33. Hilbert and Cohn-Vossen, Anschauliche Geometrie, 1932, pp. 237, 238.

Massachusetts Institute of Technology 